\title{
Novel Fiber Optic Current Transformer With New Phase Modulation Method
}

\author{
Yuefeng QI ${ }^{1,2}$, Mingjun WANG ${ }^{1 *}$, Fengxian $\mathrm{JIANG}^{3}$, Xin $\mathrm{ZHANG}^{1}$, \\ Bitong $\mathrm{CONG}^{1}$, and Yanyan $\mathrm{LIU}^{1,2}$ \\ ${ }^{1}$ School of Information Science and Engineering, Yanshan University, Qinhuangdao 066004, China \\ ${ }^{2}$ The Key Laboratory for Special Fiber and Fiber Sensor of Hebei Province, Qinhuangdao 066004, China \\ ${ }^{3}$ Li Ren College of Yanshan University, Qinhuangdao 066004, China \\ ${ }^{*}$ Corresponding author: Mingjun WANGＩ-mail: 534757970@qq.com
}

\begin{abstract}
Based on the transverse electro-optic effect of lithium niobate crystal, combined with polarizers and Faraday rotator, this paper presents a collinear closed-loop fiber optic current transformer with spatial non-reciprocity modulation method, and the feasibility of the scheme is verified by both the theoretical and experimental evidences. The detection scheme avoids the limitation of the transition time of the sensing fiber coil on the phase modulation frequency, improves the sensitivity and stability of the system, and reduces the volume and cost of fiber optic current transformer. The sawtooth wave modulation scheme is adopted to realize phase bias modulation and feedback modulation through phase shift of sawtooth wave to achieve closed-loop detection effect, which enhances the signal to noise ratio and simplifies demodulation mode. The experimental results show that the current ratio errors measured at room temperature range from $1 \%$ to $120 \%$ of rated current meet the requirements of national standard GB/T 20840.8-2007 and reach the accuracy level of $0.2 \mathrm{~S}$. The temperature stability of the current transformer is also tested, and the ratio error measured at the rated current does not exceed $\pm 0.2 \%$ in the range of $-30{ }^{\circ} \mathrm{C}$ to $50{ }^{\circ} \mathrm{C}$.
\end{abstract}

Keywords: Fiber optic current transformer; spatial phase modulation; closed-loop detection; current ratio error; temperature stability

Citation: Yuefeng QI, Mingjun WANG, Fengxian JIANG, Xin ZHANG, Bitong CONG, and Yanyan LIU, "Novel Fiber Optic Current Transformer With New Phase Modulation Method," Photonic Sensors, 2020, 10(3): 275-282.

\section{Introduction}

With the rapid development of the economy and the increasing demand for electricity, the power supply voltage level of China's transmission systems is also constantly increasing. At this stage, the country has built and put into operation a number of ultra-high voltage transmission projects. As one of the main protective and monitoring devices in the electric power system, the current transformer plays an important role in collecting and monitoring the current information in the operation of the electric power system to ensure the safety and reliability of the electricity supply. In recent years, with the rapid development of optical fiber sensing technology [1], fiber optic current transformer (FOCT) emerges as a new transformer. It adopts an all-optical fiber structure and has a series of advantages such as good insulation, small size, fine dynamic characteristics, and high stability [2]. It overcomes the problems of

Received: 21 September 2019 / Revised: 09 January 2020

(C) The Author(s) 2020. This article is published with open access at Springerlink.com

DOI: $10.1007 / \mathrm{s} 13320-020-0581-6$

Article type: Regular 
poor insulation, small dynamic range, and susceptibility to electromagnetic interference existing in traditional electromagnetic current transformers [3, 4] and adapts to the trend of digitalization and intellectualization of the power system.

Fiber optic current transformer is a kind of current sensing device based on Sagnac interferometer theory [5] and Faraday magneto-optic effect [6]. At present, most transformers use Y waveguide or piezoelectric ceramic phase modulator to realize phase modulation. The generation of bias phase and feedback phase depends on the transit time of the optical fiber coil [7]. In order to ensure the time delay required for modulation, an additional delay coil is usually added after the phase modulator, which inevitably introduces more linear birefringence caused by the stress field [8], which reduces system stability and also adds extra volume and cost. On the other hand, the mismatch between the delay coil length and the modulation circle frequency causes an error in the phase shift of the phase modulator output, which reduces the accuracy of the system [9]. During signal detection, the sampling frequency will also be affected by the transit time. A short transit time requires a high sampling frequency, which will increase the noise bandwidth of the system and make it more susceptible to interference, and bring difficulties to the application of FOCT [10].

To address the above problems, this paper introduces a new phase modulation and demodulation scheme for FOCT, and proposes a spatial non-reciprocal phase modulation method based on device characteristics. This scheme is to phase-modulate light in different axial directions at the same time and directly introduce the phase delay between the interference lights through the modulation voltage. This method avoids the limitation of the intrinsic frequency [11] to the modulation frequency and enables the system to obtain more free sensitivity and dynamic range [12].
At the same time, the phase error introduced by the extra delay coil is avoided, and the measurement accuracy of the system is improved. According to the refractive index ellipsoid theory of electro-optical crystal, the feasibility of the phase modulation method is analyzed in theory. The prototype is designed and used for current measurement. The feasibility of the scheme is verified by experiments.

\section{Working principle}

Figure 1 shows the structure of a collinear fiber optic current transformer based on the spatial nonreciprocal modulation.

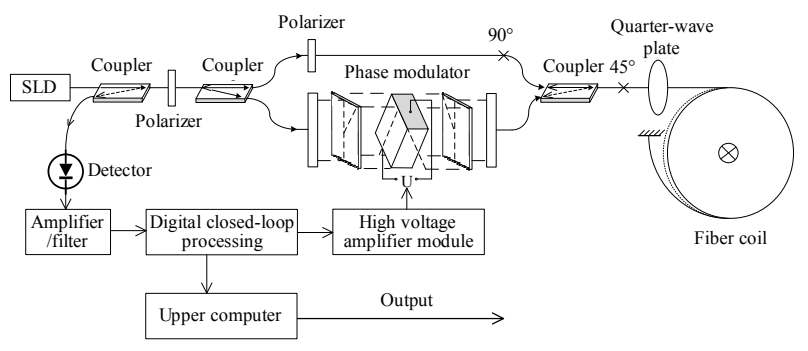

Fig. 1. Structure diagram of FOCT based on spatial phase modulation method.

Two $1 \times 2$ couplers are used between the light source and the phase modulator to satisfy the minimum nonreciprocity on the optical path. The working principle is as follows: the light emitted by the super luminescent diode light source is split into two paths of linearly polarized light after passing through the coupler. One beam of linearly polarized light passes through a $90^{\circ}$ fusion point and the other through the phase modulator. After passing through the quarter-wave plate at an angle of $45^{\circ}$, they become two circularly polarized lights with opposite rotation and enter the fiber coil. Due to the Faraday effect, the circularly polarized lights transmitted in the fiber coil rotate on the polarization plane, and then become linearly polarized light through the quarter-wave plate after being reflected back by the mirror. The linearly polarized light carrying phase information passes through the respective polarizer to eliminate the excess polarized light, and then 
enters the photodetector after interfering in the fiber coupler. Finally, digital signal processing is performed on the received light intensity signal to obtain the variation of the current.

\subsection{Faraday magneto-optical effect}

Faraday magneto-optical effect means that when plane-polarized light passes through a magnetooptical medium in the direction of the magnetic field, its polarization direction will rotate. The relationship between rotation angle $\Delta \varphi_{f}$ and magnetic field strength $H$ is given by

$$
\Delta \varphi_{f}=V \int H \mathrm{~d} l
$$

where $V$ is the Verdet constant, and $l$ is the distance traveled by light in a magneto-optical medium.

Under the Faraday effect, the phase of the circularly polarized light will change in advance or lag when it passes through the magneto-optic medium. There is a phase difference between two circularly polarized beams with different directions of rotation [13]. The optical rotation characteristic of the Faraday effect is a non-reciprocal optical process, so the total rotation angle of the forward and reverse transmission beams in fiber coil is not zero, but a superposition of two rotation angles. The intensity of the magnetic field generated around the energized wire in the fiber coil is $H=I / 2 \pi R$, where $R$ is the radius of the fiber coil, which is put into (1) to get the relationship between the tion angle and the current is

$$
\Delta \varphi_{f}=4 V N_{f} I
$$

where $N_{f}$ is the number of turns of the fiber ring, and $I$ is the current of the electrified wire. If the electrified wire is a solenoid around the fiber coil, then the above equation becomes

$$
\Delta \varphi_{f}=4 V N_{f} N_{c} I
$$

where $N_{c}$ is the turns of the electrified solenoid.

\subsection{Principle of the spatial nonreciprocal phase modulator}

The inherent response of a Sagnac type current transformer is a cosine function. When the phase difference is $\Delta \varphi_{f}=0$, the output sensitivity of the system is very small. In order to obtain the maximum output sensitivity, a phase offset of $90^{\circ}$ is often introduced to the system by an additional phase modulator. At the same time, this phase modulator introduces a feedback phase of $-\Delta \varphi_{f}$, which makes the system work at the most sensitive output point all the time [14].

The structure of the spatial nonreciprocal phase modulator is shown in Fig. 2, which is composed of polarizers, Faraday rotators, and a lithium niobate crystal. The modulation scheme of lithium niobate crystal is $y$-direction voltage and $z$-direction light transmission. The forward transmitted linearly polarized light travels along the $y$-axis of lithium niobate crystal through the Faraday rotator rotating $45^{\circ}$ clockwise $\mathrm{F}_{\mathrm{cw}}$. The reversely transmitted linearly polarized light travels along the $x$-axis of lithium niobate crystal through the faraday rotator rotating $45^{\circ}$ anticlockwise $\mathrm{F}_{\mathrm{ccw}}$. The phase difference $\Delta \varphi_{m}$ between the two beams is given by

$$
\Delta \varphi_{m}=\frac{2 \pi}{\lambda}\left(n_{x}-n_{y}\right) l
$$

where $\lambda$ is the wavelength of the light source, $n_{x}$ and $n_{y}$ are the refractive index of the crystal $x$-axis and $y$-axis, and $l$ is the length of the crystal $z$-axis.

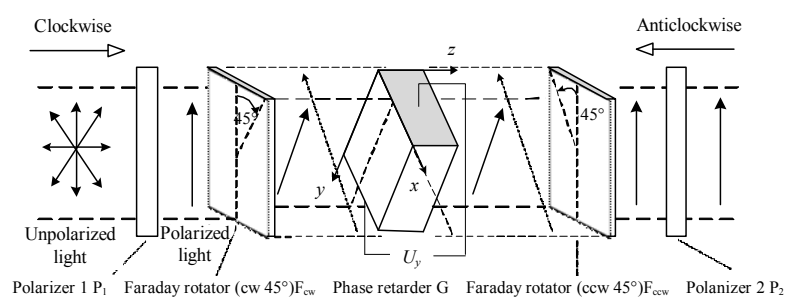

Fig. 2. Structural diagram of spatial nonreciprocal phase modulator.

Based on the primary electro-optic effect in lithium niobate crystal, the axial refractive index will change under the action of the external electric field, and the refractive index change of the induced optical axis is proportional to the first power of the applied electric field. Therefore, the nonreciprocal phase shift can be produced by controlling the electric field intensity to change the refractive index 
difference between the two induced optical axes. A transverse electro-optic modulation [15] with electric field direction perpendicular to the direction of light propagation is applied to lithium niobate crystal shown as the voltage $U_{y}$ in Fig. 2. The ellipsoid of refractive index of the crystal can be expressed as follows:

$$
\left(\frac{1}{n_{o}^{2}}-\gamma_{22} E_{y}\right) x^{2}+\left(\frac{1}{n_{o}^{2}}+\gamma_{22} E_{y}\right) y^{2}+\left(\frac{1}{n_{e}^{2}}\right) z^{2}=1 \text {. }
$$

where $n_{o}$ is the ordinary refractive index, $n_{e}$ is the extraordinary refractive index, and $E_{y}$ is the electric field intensity in the $y$-axis direction of the crystal. Based on the above analysis, we can obtain

$$
\left\{\begin{array}{l}
n_{x}=n_{o}+\frac{1}{2} n_{o}^{3} \gamma_{22} E_{y} \\
n_{y}=n_{o}-\frac{1}{2} n_{o}^{3} \gamma_{22} E_{y}
\end{array}\right.
$$

Substituting (6) into (4) yields the phase delay of the phase modulator as

$$
\Delta \varphi_{m}==\frac{2 \pi}{\lambda} n_{o}^{3} \gamma_{22} U_{y} \frac{l}{d}
$$

where $\gamma_{22}$ is the electro-optic coefficient tensor of the lithium niobate crystal, and $d$ is the thickness in the $y$-axis direction of the crystal.

It can be seen from (7) when the wavelength of the input light source and the size of the lithium niobate crystal are determined, the phase delay generated by the phase modulator is only related to the voltage applied at both ends of the crystal, and does not depend on the transit time of the fiber coil.

\subsection{Principle of light intensity signal demodulation}

According to the closed-loop detection principle of fiber optic gyroscope, a closed-loop detection scheme of fiber optic current transformer based on the spatial phase modulation method is proposed. As shown in the structure of the collinear current transformer shown in Fig. 1, the detected interference light intensity $I_{d}(t)$ can be expressed as follows:

$$
I_{d}(t)=I_{o}\left[1+\cos \left(\Delta \varphi_{m}+\Delta \varphi_{f}\right)\right]
$$

where $I_{o}$ indicates the peak intensity, which is related to the light output intensity and transmission loss.

The closed-loop method of spatial phase modulator is different from the first harmonic detection of piezoelectric ceramics or the digital step wave detection of $\mathrm{Y}$ waveguide, which adopts sawtooth wave modulation, as shown in Fig. 3(a). The modulation phase shift of the optical signal generated by the phase modulator is $2 \pi t / T-k \pi$, where $T$ is the period of the modulation signal, and $k$ is an even number. $2 \pi / T$ is represented by circular frequency $\omega_{m}$, and the interference optical intensity signal is

$$
I_{d}(t)=I_{o}\left[1+\cos \left(\omega_{m} t-k \pi+\Delta \varphi_{f}\right)\right] .
$$

In the sawtooth wave signal shown in Fig.3(a), $k$ is an even number, and (9) can be simplified to

$$
I_{d}(t)=I_{o}\left[1+\cos \left(\omega_{m} t+\Delta \varphi_{f}\right)\right] .
$$

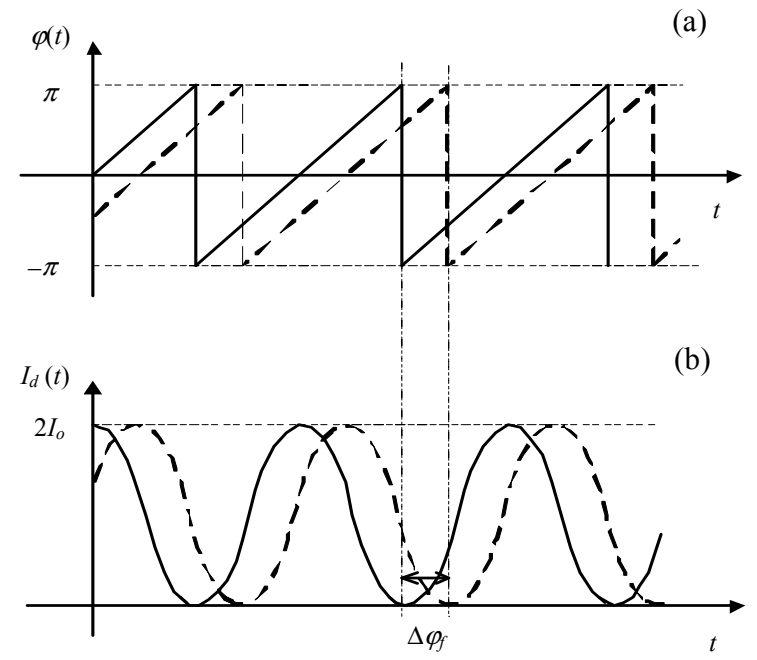

Fig. 3 Relationship between the modulated signal and the detected light intensity.

The corresponding relationship between the modulation phase shift and the interference light intensity is as shown in Fig. 3. When the modulation signal produces a phase shift of a certain angle, the interference light intensity signal also undergoes a corresponding angle translation. Therefore, the $\pi / 2$ translation phase can be generated by controlling the modulation voltage of the phase modulator, so that 
the cosine signal can be converted into sinusoidal signal to achieve the purpose of bias modulation. When the Faraday phase shift of $\Delta \varphi_{f}$ is detected, an additional feedback phase shift $-\Delta \varphi_{f}$ is generated to achieve closed-loop feedback modulation, so that the current transformer always works at the working point with the maximum detection sensitivity. It can be seen from (10) that the Faraday phase shift can be obtained by Fourier transform of light intensity signal, and then the corresponding current to be measured can be obtained.

\section{Experimental measurements and results analysis}

In order to verify this scheme, a prototype combined National Instruments data acquisition card [16] to sample and demodulate the detection signal is designed to measure the primary current and the temperature stability of FOCT. Figure 4 shows the physical diagram of the principle prototype.

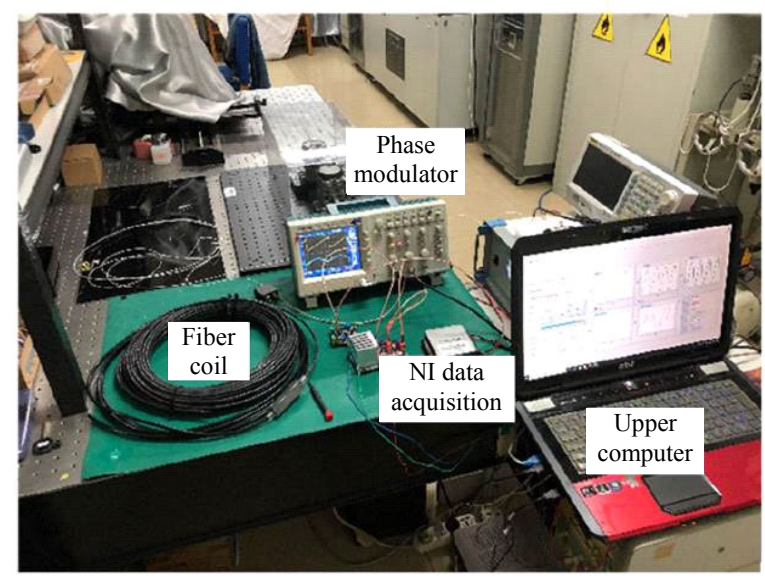

Fig. 4 Physical diagram of the prototype.

\subsection{Primary current measurement experiments}

In the experimental prototype, the fiber coil is winded with circular polarization-maintaining fiber for 40 turns, and the rated current of the current transformer prototype is $1500 \mathrm{~A}$. The sampling frequency of the prototype is $5 \mathrm{kHz}$ in the experiment. Due to the influence of system error, the actual variable ratio of the system should be calculated before measuring the current for calibration. The actual ratio is the ratio of the root mean square of the actual primary current to the root mean square of the secondary current. The actual measured ratio of the prototype is 4.363323 .

A total of 20 experiments were performed at room temperature. In addition to small currents of $15 \mathrm{~A}$ and $75 \mathrm{~A}$, the current data were recorded every $100 \mathrm{~A}$ from the range of $15 \mathrm{~A}$ to $1800 \mathrm{~A}$. The data recorded in the experiment were the current data derived from LabVIEW upper computer program. Figure 5 shows the measurement diagram of FOCT with the primary current of $75 \mathrm{~A}(5 \%), 300 \mathrm{~A}(20 \%)$, $1500 \mathrm{~A}(100 \%)$, and $1800 \mathrm{~A}(120 \%)$, respectively.

As can be seen from Fig. 5, the larger the primary current is, the smaller the noise variation amplitude in the detected waveform is, and the smaller the ratio of the peak-to-peak value of the noise to the measured current is, the higher the accuracy is. In the whole FOCT, the noise does not decrease with the change of the primary current. Therefore, it can be determined that the detection noise in the optical path increases with the current decrease, which is in line with the characteristics of the FOCT suitable for large current measurement. For the prototype designed in this paper, it should mainly conform to the relevant requirements of national standard GB/T 20840.8-2007, that is, the response time does not exceed $5 \mathrm{~ms}$, and the accurate level should satisfy the error limit of current transformer for special purpose. In the four groups of detection waveforms shown in Fig. 5, the response time is $2 \mathrm{~ms}-3 \mathrm{~ms}$, which meets the requirements of relay protection.

The scatter diagram is drawn and linearly fitted for 20 groups of measured current data, as shown in Fig. 6. Each discrete point in the figure is the average value of the secondary current. The linear regression coefficient of the fitting curve of the transformer output is $R^{2}=1$, the linear slope is 0.99989 , and the zero deviation is $2.617 \times 10^{-4}$. It indicates that the prototype used in the test has good linearity. 


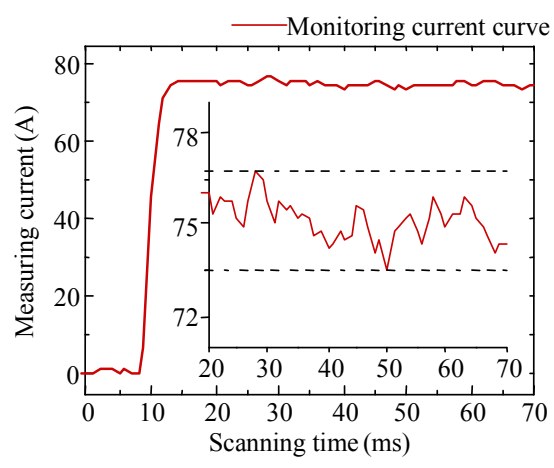

(a)

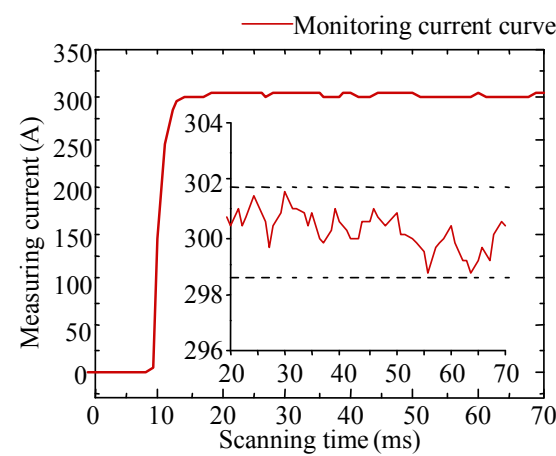

(b)

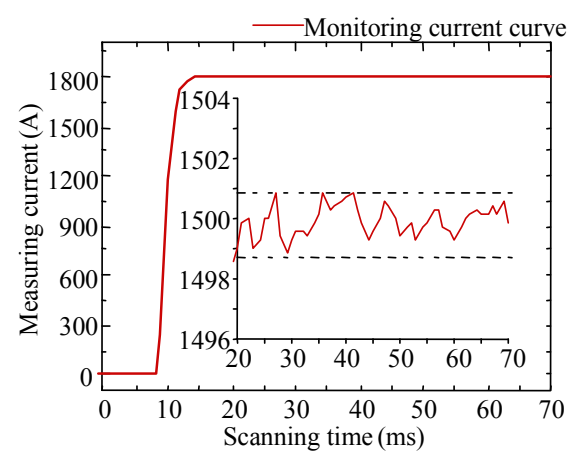

(c)

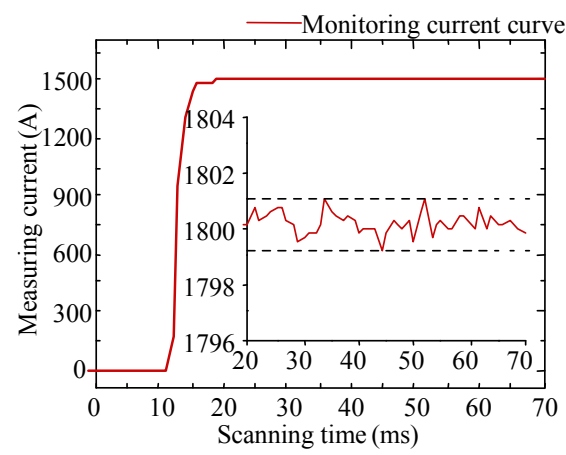

(d)

Fig. 5 Current variation diagram of the prototype: (a) the primary current is $75 \mathrm{~A}$, (b) the primary current is $300 \mathrm{~A}$, (c) the primary current is $1500 \mathrm{~A}$, and (d) the primary current is $1800 \mathrm{~A}$.

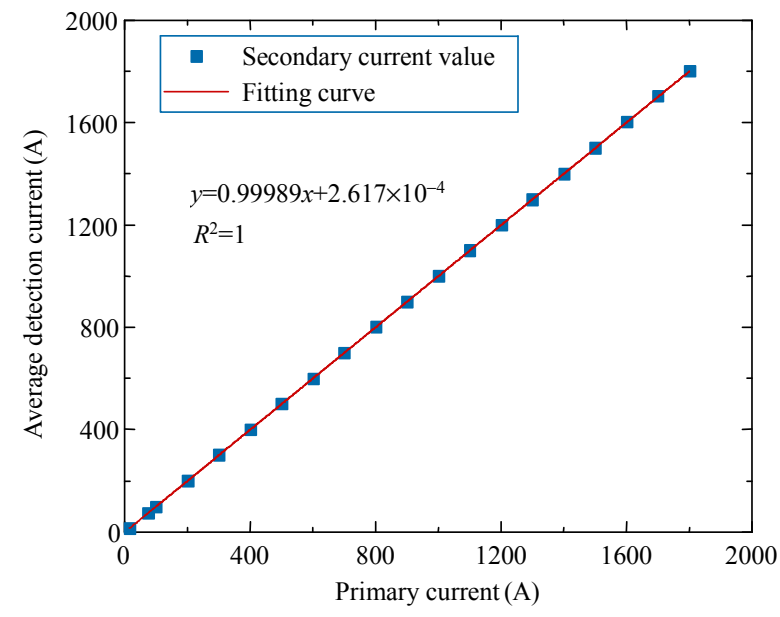

Fig. 6 Output linearity of FOCT.

According to the national standard GB/T 20840.8-2007, the current transformer ratio error [17] is defined as

$$
\mathcal{E}=\frac{K_{\mathrm{ra}} \times I_{s}-I_{p}}{I_{p}} \times 100 \%
$$

where $K_{\mathrm{ra}}$ is the actual transformation ratio, $I_{s}$ is the root mean square value of the secondary current, and $I_{p}$ is the root mean square value of the primary current.

At room temperature, the input current is $1 \%$, $5 \%, 20 \%, 100 \%$, and $120 \%$ of the rated current of $1500 \mathrm{~A}$, respectively. The ratio error of the current transformer is compared with the $0.2 \mathrm{~S}$ level stipulated by the national standard, as shown in Table 1.

Table 1 FOCT ratio error table.

\begin{tabular}{ccc}
\hline $\begin{array}{c}\text { Percentage of rated } \\
\text { current }\end{array}$ & $\begin{array}{c}0.2 \mathrm{~S} \text { standard ratio } \\
\text { error } /( \pm \%)\end{array}$ & $\begin{array}{c}\text { Prototype ratio error } \\
(\%)\end{array}$ \\
\hline $1 \%$ & 0.75 & 0.55467 \\
$5 \%$ & 0.50 & -0.30292 \\
$20 \%$ & 0.20 & 0.06061 \\
$100 \%$ & 0.20 & 0.04907 \\
$120 \%$ & 0.20 & 0.05288 \\
\hline
\end{tabular}

It can be seen from Table 1 that the current transformer using this modulation and demodulation scheme conforms to the requirements of $0.2 \mathrm{~S}$ level special purpose current transformers specified in national standard GB/T 20840.8-2007 at room temperature. 


\subsection{Temperature stability experiment}

The measurement accuracy of the experimental prototype machine reaches $0.2 \mathrm{~S}$ level at room temperature. However, both the fiber coil and the quarter wave plate are placed in the outdoor environment for practical applications. The polarization state of the optical signal is affected by the environment temperature. Therefore, the performance index of FOCT under the full temperature state, especially the measurement accuracy, also needs to meet the requirements.

After calibrating the FOCT at room temperature, the sensor coil is placed in the temperaturecontrolled cabinet to measure the change of its ratio error at different temperatures. The experimental temperature ranged from $-40^{\circ} \mathrm{C}$ to $80^{\circ} \mathrm{C}$, and the data were recorded every $10^{\circ} \mathrm{C}$. After reaching the set temperature, the sensor coil was heated evenly for $15 \mathrm{~min}$. Then the rated current of $1500 \mathrm{~A}$ was applied to the FOCT to record the secondary current and calculate the ratio error.

The ratio error of the FOCT varies with temperature as shown in Fig. 7. It can be seen from the figure that the ratio error of the FOCT is within $\pm 0.2 \%$ in the range of $-30^{\circ} \mathrm{C}$ to $50^{\circ} \mathrm{C}$, which can conform to the measurement requirement of $0.2 \mathrm{~S}$ level.

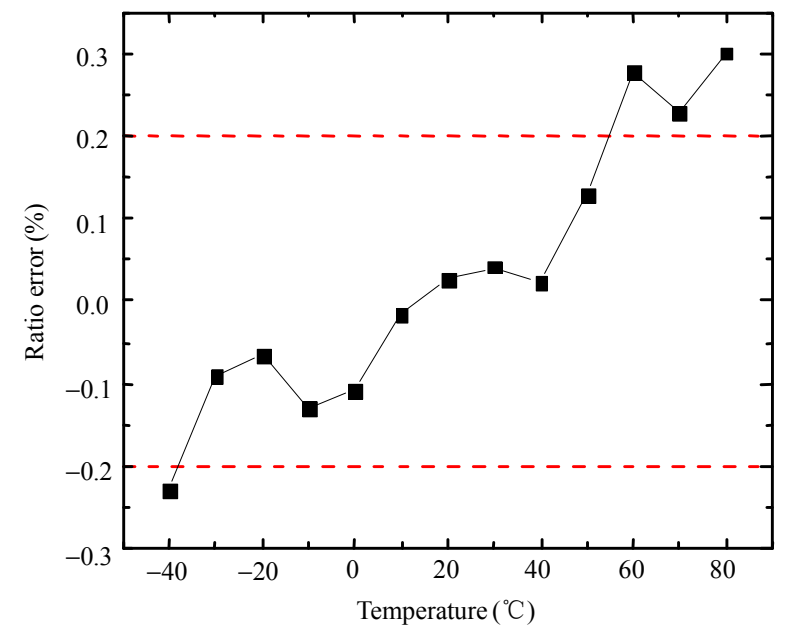

Fig. 7 Test diagram of FOCT ratio error with temperature variation

The main factors affecting the measurement accuracy of the transformer during the experiment are the Verdet constant of the sensing fiber and the phase delay of the quarter-wave plate. When the temperature changes, the Verdet constant will increase with an increase in temperature, which will cause additional phase difference between two polarized lights in the sensing fiber and introduce detection error. The change of phase delay angle of quarter-wave plate will change the polarization of optical signal and affect the detection accuracy of the system.

\section{Conclusions}

A new phase modulation and demodulation scheme for optical fiber current transformer is described in detail. The bias modulation and feedback modulation of the transformer are realized by using the primary electro-optical effect of the uniaxial crystal to change the refractive index difference between the optical axes. The phase modulator uses sawtooth wave signal as the modulation voltage. Fourier transform is performed on the interfering optical signal to demodulate the Faraday phase shift. The phase modulation method in this scheme does not depend on the delay time of the fiber coil, which avoids the limitation of the modulation frequency by the intrinsic frequency. At the same time, phase demodulation is also easier to implement. The prototype of the FOCT is built in order to verify the reliability of the theory, and the main performance of the prototype is tested. The experimental results show that under the condition of room temperature, the current transformer of the scheme satisfies the error limitation of the national standard at the rated current of $1 \%-120 \%$, and achieves the accuracy of $0.2 \mathrm{~S}$ level. In the temperature range of $-30^{\circ} \mathrm{C}$ to $50^{\circ} \mathrm{C}$, the ratio error measured under the rated current does not exceed $\pm 0.2 \%$.

\section{Acknowledgment}

This work was supported by the National Natural Science Foundation of China (Grant No. 
61735011), the Science and the Key Research and Development Program of Hebei Province (Grant No. 19251703D), and the Local Science and Technology Development Projects of the Central Committee (Grant No. 19941708G).

Open Access This article is distributed under the terms of the Creative Commons Attribution 4.0 International License (http://creativecommons.org/licenses/by/4.0/), which permits unrestricted use, distribution, and reproduction in any medium, provided you give appropriate credit to the original author(s) and the source, provide a link to the Creative Commons license, and indicate if changes were made.

\section{References}

[1] B. Lee, "Review of the present status of optical fiber sensors," Optical Fiber Technology: Materials, Devices and Systems, 2003, 9(2): 57-79.

[2] K. Bohnert, P. Gabus, J. Kostovic, and H. Brandle, "Optical fiber sensors for the electric power industry," Optics and Lasers in Engineering, 2005, 43(3/4/5): 511-526.

[3] X. K. Song, P. L. Yan, Z. H. Xiao, D. W. Liu, and Y. B. Liu, "Comment on the technology and application of fiber optic current transformer," Power System Protection and Control, 2016, 44(8): 149-154.

[4] X. X. Wang, C. X. Zhang, C. Y. Zhang, and Z. J. Wu, "A new all digital closed-loop fiber optic current transformer," Automation of Electric Power Systems, 2006, 30(16): 77-80.

[5] B. Culshaw, "The optical fibre Sagnac interferometer: an overview of its principles and applications," Measurement Science \& Technology, 2005, 17(1): R1.

[6] A. Ghosh, P. B. D. Gupta, and A. K. Mandal, "Development of a fiber-optic current sensor with range-changing facility using shunt configuration," IEEE Sensors Journal, 2013, 13(4): 1347-1354.

[7] Y. F. Qi, Q. Feng, J. Zhang, X. Zhang, M. J. Wang, D. S. Tian, et al., "Design of fiber-optic current transformer based on passive phase modulator," Acta Optica Sinica, 2019, 39(4): 0406004.

[8] Y. Liu, J. S. Xia, and Y. Zhang, "Problem of linear birefringence effect in optical current transducer and its countermeasure," Journal of Transducer Technology, 2000, 19(3): 11-14.

[9] S. Sun, Z. P. Wang, and Y. K. Wang, "Effect of delayline length on the all-fiber optic current transformer," Optics \& Optoelectronic Technology, 2013, 11(2): 48-51.

[10] V. H. Ortiz, J. L. Flores, and G. García-Torales, “A review on optical current transducers for power system metering," Proceedings of SPIE, 2007, doi: $10.1117 / 12.735082$.

[11] X. Wang, X. X. Wang, J. Yu, Y. Zhang, T. T. Wang, and F. Ma, "Eigen frequency tracking measurement of fiber optic gyroscope based on sawtooth wave modulation," Chinese Journal of Lasers, 2017, 44(12): 153-158.

[12] Z. H. Xiao, S. Cheng, G. Q. Zhang, Z. Z. Guo, P. L. Yan, and W. B. Yu, "Research on sensitivity characteristic of fiber optic current transformer," Electric Power Automation Equipment, 2017, 37(1): 212-216.

[13] Y. C. Zhong, J. Zhou, and S. H. Chen, "Study on measuring the direction of current based on the Faraday magneto-optic effect," Optical Technique, 2016, 42(1): 78-80.

[14] X. Y. Li, J. H. Hao, H. R. Yang, J. W. Yang, H. Zhou, Y. T. We, et al., "Research and development of sagnac ringlike optic current sensor," Opto-Electronic Engineering, 2011, 38(7): 1-6.

[15] K. W. Li, Z. B. Wang, R. Zhang, G. L. Wang, and Y. L. Wang, " $\mathrm{LiNbO}_{3}$ transverse electro-optical modulation characteristics for light pass along optical axis," Optics and Precision Engineering, 2015, 23(05): 1227-1232.

[16] X. S. Liu, W. B. Xiao, H. M. Wu, Y. S. Xiao, and L. Z. Huang, "Design of integrated testing system for optical fiber current transformer based on labview," Computer Measurement \& Control, 2017, 25(7): $35-42$.

[17] T. T. Hu, X. C. Ma, and C. Y. Zhang, "Calibration of the accuracy for all fiber-optical current transducer," High Voltage Apparatus, 2012, 48(5): 80-83. 